\title{
Affine invariant contour matching using a voting approach
}

\author{
Wei Wang, Xingwei Yan, Yongmei Jiang, Gangyao Kuang \\ School of Electronic Science and Engineering \\ NUDT \\ Changsha, China \\ Wangwei8610@gmail.com
}

\begin{abstract}
By observing the descriptors of the contour points, correspondence based contour matching is widely used in pattern recognition. However, it is a pity that the correspondences established by descriptor matching are not reliably. In this paper, a matching approach, in which the inlier correspondences in matched configurations are used for voting, is proposed. Experimental results show that the proposed voting approach is well suited for contour point matching under affine transformation and noise.
\end{abstract}

Keywords-affine, moment, point matching, voting

\section{INTRODUCTION}

Contour matching is an important task in computer vision such as image analysis and pattern recognition. However, despite great effort, it is still a difficult problem especially when the contours are noise disturbed or distorted due to different viewpoint. It is well known that the deformations between shapes can be approximated by affine transformations if the viewpoints are far enough. Consequently, invariant to affine transformation is a desirable property for many contour matching problems. The contours, which are originally defined by $\mathrm{x}$ and $\mathrm{y}$ coordinates of points, contain considerable amount of information, and a number of contour matching methods have been suggested for contour recognition under affine transformation.

Contour matching methods can be generally divided into two classes: feature-based methods [1-4] and correspondence-based methods. The classification is based on whether the correspondences between points are taken into account in contour matching. In the feature-based methods, such as shape signatures, curvature scale space, and $\mathrm{R}$-histogram et al, the features are extracted as descriptors to measure the similarity between contours. In contrast to the feature-based methods, correspondence-based methods find the matched contours based on point-to-point matching.

Hausdorff distance [5] is a classical correspondencebased method, in which Euclidean distance is used to locate contours. Belongie et al. recover the correspondences between two boundary point sets based on the shape context (SC) [6,7], which describes the distribution of the rest of the contour with respect to a given point on the contour.

Moment, which can be treated as the description for the point, is one of the widely used technique works for point-topoint matching. One of the most widely used contour moment is FD [8], and has been extended to be affine invariant by Arbter et al. Yang and Cohen used a new set of cross-weighted moments to analytically solve for point matching. Liu et al proposed an generating function to derive various affine invariant moments. Wang et al [9] taken the diagonals of the orthogonal projection matrices are the contour moments. Once the cost of moment matching has been measured, the approach for point matching shall be followed. Generally, the matched points are considered to be the ones with the minimal matching cost. However, to subject to the constraint that the matching be one-to-one, dynamic programming (i.e. Hungarian) is always adopted. Though the 'dummy' nodes are added to point sets with a constant matching cost to have robust handling of outliers, there still exist outlier in the correspondences obtained by the Hungarian method. Therefore, to further improve the accuracy of point matching, a useful schema of outlier removal based on the monotonic property of contour correspondence has been proposed in [9]. The monotonic property implies that the ordering of points matching should be monotonic regardless of a specific indexing of the points.

Motivated by [9], to remove the outliers in the correspondences, a novel point matching algorithm based on voting is suggested. As we known, since the moment is extracted from the configuration of the point, the matching of two point are actually the matching of two local configurations. Accordingly, the points in the configurations of two matched points are inlier correspondences. Then, the correspondences between configurations of two matched points can be used to validate the correspondence between the two points.

The rest of the paper is organized as the follows. Section 2 describes a general system for point matching. The voting schema for outlier removal is suggested in Section 3. The experimental examples are given in Section 4, and Section 5 concluded the paper.

\section{CONTOUR RECOGNITION BASED ON POINT-TO-POINT MATCHING}

The contour recognition system usually includes two stages: descriptor extracting and point matching. In this section, we take the DOPM moment as an example to introduce the point matching system.

As shown in Fig.1, for the point $p_{i}$ on the contour $E$, we pick up $2 n$ points in its neighborhood to form a local configuration $X_{p}=\left[p_{i-n}, \ldots, p_{i}, \ldots, p_{i+n}\right]^{T} \in R^{n \times 2}$, and an augmented configuration matrix is defined as $\bar{X}_{p}=\left[X_{p}, 1_{2 n+1}\right] \in R^{(2 n+1) \times 3}$, where $1_{2 n+1}=[1,1, \ldots, 1] \in R^{2 n+1}$, 
where $1_{2 n+1}=[1,1, \ldots, 1] \in R^{2 n+1}$. Then, the orthogonal projection matrix of $p$ is

$$
\Pi_{p}=\bar{X}_{p}\left(\bar{X}_{p}^{T} \bar{X}_{p}\right)^{-1} \bar{X}_{p}^{T}
$$

Let a general 2-D affine transformation $T$ transforms the configuration $X_{p}$ into $Y_{q}$ by

$$
\bar{Y}_{q}=\bar{X}_{p} T=\bar{X}_{p}\left[\begin{array}{ll}
A_{T} & 0 \\
b & 1
\end{array}\right],
$$

where $b_{2 \times 1}$ is the translation vector and $A_{T 2 \times 2}$ is the affine transformation matrix between the two images. Rotation, scaling and shearing, considered as the special cases of affine transform, are represented as the following matrices:

$$
\begin{aligned}
& A_{\text {T scaling }}=\left(\begin{array}{ll}
s_{x} & 0 \\
0 & s_{y}
\end{array}\right), A_{\text {Trotation }}=\left(\begin{array}{rr}
\cos \theta & -\sin \theta \\
\sin \theta & \cos \theta
\end{array}\right), \\
& A_{\text {T shearing }}=\left(\begin{array}{ll}
1 & k \\
0 & 1
\end{array}\right)
\end{aligned}
$$

Assume the points used to form the configuration are extracted from the contours in order, therefor, we have

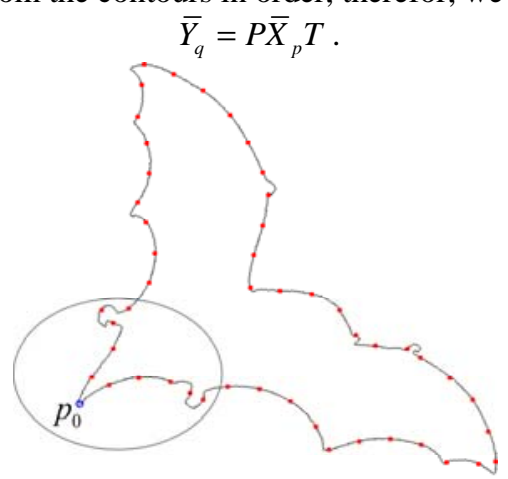

(a)

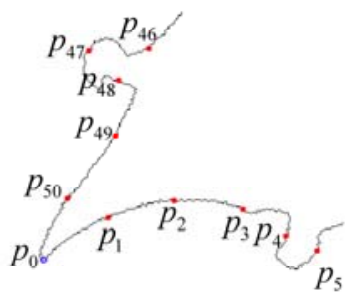

(b)

Figure 1. Example of the configuration of point $p_{0}$. (a) The sampled points on the contour. (b) The neighboring points for $p_{0}$ are picked up to form the configuration of $p_{0}$.

In which, the permutation matrix $P$ can be simplified to the identity matrix $I$ or the reverse-identity matrix $J$, i.e.,

$$
I=\left[\begin{array}{lll}
1 & & 0 \\
& \ddots & \\
0 & & 1
\end{array}\right], \quad J=\left[\begin{array}{lll}
0 & & 1 \\
& . & \\
1 & & 0
\end{array}\right]
$$

In application of (1), the orthogonal projection matrix of $q$ is

$$
\begin{aligned}
\Pi_{q} & =\bar{Y}_{q}\left(\bar{Y}_{q}^{T} \bar{Y}_{q}\right)^{-1} \bar{Y}_{q}^{T} \\
& =P \bar{X}_{p} T\left[\left(P \bar{X}_{p} T\right)^{T}\left(P \bar{X}_{p} T\right)\right]^{-1}\left(P \bar{X}_{p} T\right)^{T}=P \prod_{p} P^{T}
\end{aligned}
$$

According to (6), we can obtain

$$
\operatorname{diag}\left(\Pi_{q}\right)=P \operatorname{diag}\left(\Pi_{p}\right)
$$

So far, the DOPM moment has been proven to be affine invariant and can be taken as the descriptor of $p$.

Once the descriptor has been computed, a widely used way for point matching is based on the minimal descriptor matching error. Then, a criteria to measure the similarity between two points is defined as

$$
C(p, q)=\left\|\operatorname{diag}\left(\Pi_{p}\right)^{1 / 2}-\operatorname{diag}\left(\Pi_{q}\right)^{1 / 2}\right\|_{1} .
$$

Where, $\|.\|_{1}$ denotes the 1-norm of the vector. Given the set of costs $C_{p q}$ between all pairs of point $p$ on the first contour and $q$ on the second contour, the simplest way to compute the mapping $\bar{\rho}$ between two point sets $M_{X}$ and $M_{Y}$ is

$$
\bar{\rho}=\left\{<i, j>\in M_{X} \times M_{Y}: C_{i j} \leq \min _{<k, l>\in M_{X} \times M_{Y}}\left(C_{i l}, C_{k j}\right)\right\} .
$$

\section{OUTLIER REMOVAL}

In real application, the matched shape pair in dependence on a single corresponding descriptor pair is sometimes not reliable, as the presence of noise, and/or because of the inaccuracy in shape extracting. Therefore, there may exist outliers in the point correspondences $\bar{\rho} \subseteq M_{X} \times M_{Y}$, and some methods have been proposed to remove the outliers. For example, in [9], the ordering property of the contour is adopted to as the constraint. However, this method is effective only with the prerequisite that the points in configurations are chosen with the same order. To solve this problem, a voting approach is suggested for outlier removal.

For a matched point pair $\left(p_{i}, q_{j}\right)$, the points in their configurations $\left\{p_{i-n}, \ldots, p_{i}, \ldots, p_{i+n}\right\}$ and $\left\{q_{j-n}, \ldots, q_{j}, \ldots, q_{j+n}\right\}$ are considered to be inlier correspondences, since the matching of two points are actually the matching of two local configurations. In other words, we have

$$
\begin{aligned}
& p_{i} \Leftrightarrow q_{j} \Rightarrow\left\{p_{i-n}, \ldots, p_{i}, \ldots, p_{i+n}\right\} \Leftrightarrow \\
& \left\{q_{j-n}, \ldots, q_{j}, \ldots, q_{j+n}\right\} \Rightarrow p_{i-m} \Leftrightarrow q_{j-m} \quad m \in[-n, n]
\end{aligned}
$$

where, ' $\Leftrightarrow$ ' denotes the correspondence relationship. Extended by (10), $\forall m \in[-n, n]$, we get

$$
\begin{aligned}
& p_{i-m} \Leftrightarrow q_{j-m} \Rightarrow\left\{p_{i-m-n}, \ldots, p_{i-m}, \ldots, p_{i-m+n}\right\} \\
& \Leftrightarrow\left\{q_{j-m-n}, \ldots, q_{j-m}, \ldots, q_{j-m+n}\right\} \Rightarrow p_{i} \Leftrightarrow q_{j} .
\end{aligned}
$$

Equation (11) implies that, besides the descriptor matching, the the matched point pair $\left(p_{i}, q_{j}\right)$ also can be established by the correspondences $\left(p_{i-m}, q_{j-m}\right)$ when $m \in[-n, n]$. Thus, to improve the accuracy of point matching, a voting approach is proposed. In the following step, we show how to find the matched point for the point $p_{i}$ : 
Step 1: All candidate correspondences are obtained by descriptor matching using (9);

Step 2: For each candidate matched point pair $\left(p_{i}, q_{j}\right)$, it is casted a vote. In addition, the inliers correspondences $\left(p_{i-m}, q_{j-m}\right)$, where $m \in[-n, n]$, are all casted a vote;

Step 3: The candidate matched point pairs whose vote are larger than a threshold are considered as the true correspondences.

\section{EXPERIMENTAL RESULTS}

In the following, the proposed voting approach for point matching under affine transformation and noise is tested, and compared with the ordering schema. We experimented on the MPEG-7 CE shape-1 database, which contains 1400 shape images. The transformed images are obtained according to different affine transformations and various noise levels. As the general affine transformation contains scaling, rotation and shearing, it is feasible to apply only one of these transformations at a time. In other words, during each group of experiment, only one transformation parameter is changed while others are fixed. In experiments,

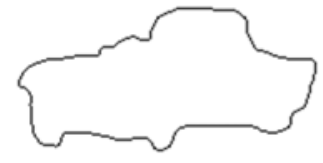

(a)

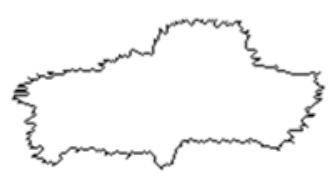

(b)

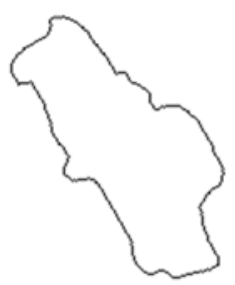

(c)

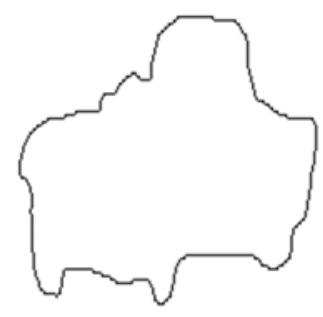

(d)

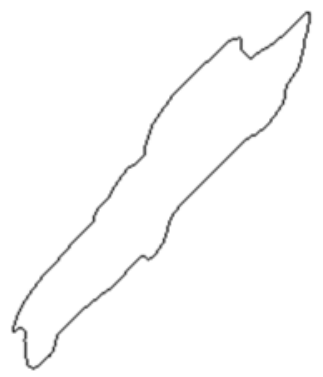

(e)
Figure 2. Example of the contours used for experiments. (a) original shape. (b) The shape obtained by adding noise with $S N R=30 \mathrm{~dB}$. (c) the shape rotated by $60^{\circ}$. (d) The shape transformed by nonuniform scaling (e) the shape transformed by shearing with $k=-1$. The shearing also contributes to the shape distortion

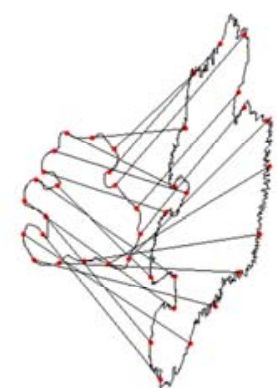

(a)

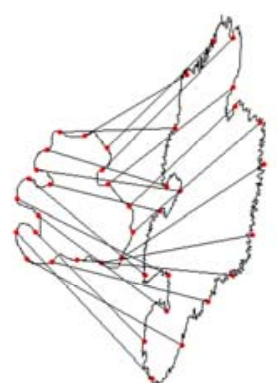

(b)

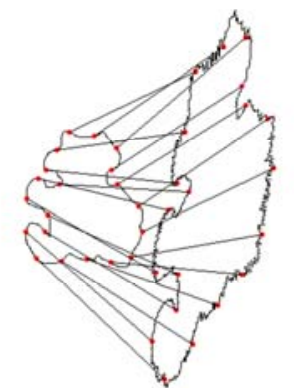

(c)

Figure 3. Results of the contour point matching using the methods of (a) minimal descriptor matching error, (b) ordering and (c) voting.

The examples of contours used in the experiments are given in Fig. 2. Fig. 3 shows results of point matching in application of minimal descriptor matching error, ordering and voting respectively when the contours are sampled by 20 points. In experiments, all contours are extracted and sampled by 100 points.

First, the original contours are rotated while the angle of rotation changes from 20 to 180, with 20 intervals, and we evaluated the effect of rotation on point matching using the proposed voting approach. Fig. 4a shows that, we get the highest accuracy of point matching using the voting approach.

Then, the performance of voting approach on point matching is tested while the contours are scaled. The value of non-uniform scaling $s y / s X$ changes from 1.2 to 3 in step of 0.2 . Fig. 4b implies that the voting approach has obvious advantages over other two methods.

In addition, the performance of point matching method with respect to shearing is summarized in Fig. 4c, depicting that the voting approach outperforms to other methods.

Finally, to compare the effect of the noise on point matching methods, the method in [5] is used for noise simulation on shapes. Fig. 4d indicates that the accuracy of point matching increases as the signal-to-noise ratio (SNR) increases, and the performance of the voting approach is the best.

\section{CONCLUSION}

In this paper, a point matching approach based on voting is proposed. After computing the descriptors from the configurations of the points, the candidate matched point 


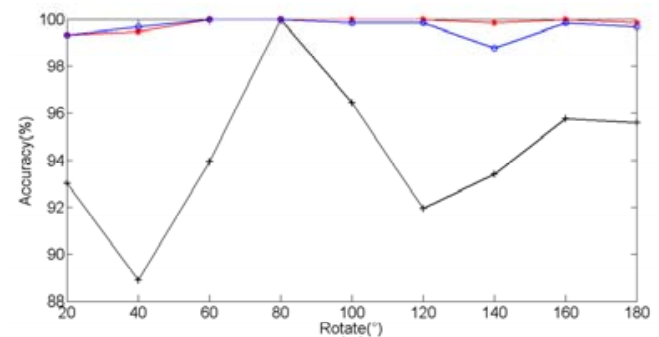

(a)

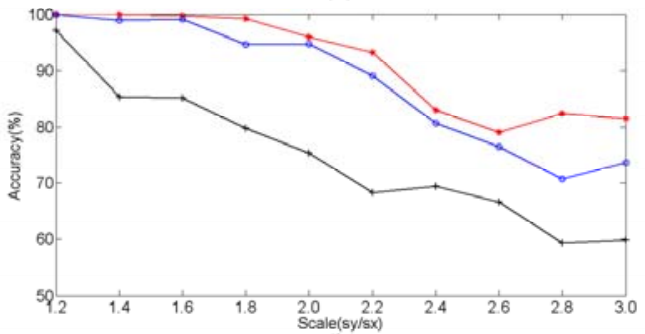

(b)

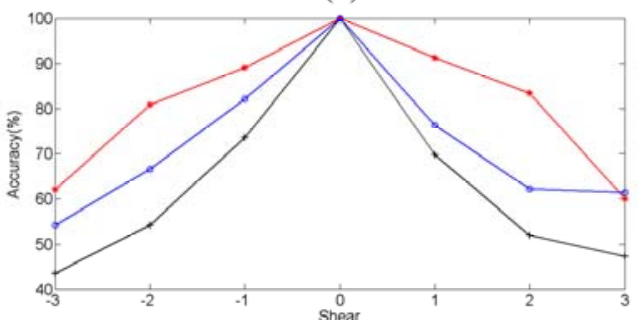

(c)

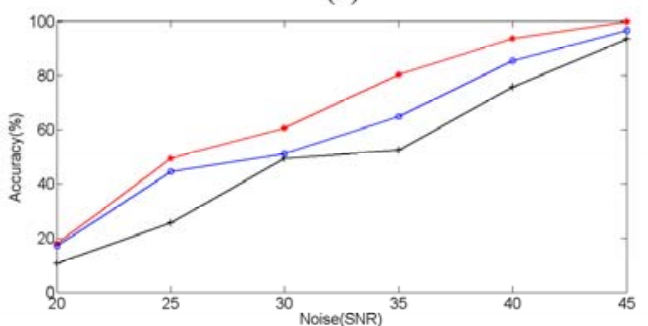

(d)

pairs are obtained by descriptor matching. Since, the points in the matched configurations are inlier correspondences, to improve the point matching accuracy, they are used for voting to established the final matched point pairs. Experimental results show that the voting approach is quite effective for point matching as regards rotation, scale, shearing and noise.

\section{ACKNOWLEDGMENT}

We thank the National Natural Science Foundation of China under Grant 61201338 and Grant 61171135.

\section{REFERENCES}

[1] J. M. K. Pascal Matsakis, Ozy Sjahputera, and Jonathon Marjamaa, The use of force histograms for affine invariant relatiove position description, IEEE Transactions on Pattern Analysis and Machine Intelligence, vol. 26, pp. 1-17, 2004.

[2] W. Wang, B. Xiong, H. Sun, et al., An affine invaraint relative attitude relationship descriptor for shape matching based on ratio histograms, EURASIP Journal on Advances in Signal Processing, vol. 2012:209, 2012.

[3] S. A. Farzin Mokhtarian, Shape similarity retrieval under afine transforms, Pattern Recognition, vol. 35, pp. 31-41, 2002.

[4] C. Q. C. F. Mai, and Y.S. Hung, "Affine-Invariant Shape Matching and Recognition under Partial Occlusion," in Proceedings of 2010 IEEE 17th International Conference on Image Processing, ed. Hong Kong, 2010, pp. 4605-4608.

[5] D. P. Huttenlocher, G. A. Klanderman and W. J. Rucklidge, comparing images using hausdorff distance, IEEE Transactions on Pattern Analysis and Machine Intelligence, vol. 15, pp. 850-863, 1993

[6] J. M. Serge Belongie, and Jan Puzicha, Shape Matching and Object Recognition using Shape Contexts, IEEE Transactions on Pattern Analysis and Machine Intelligence, vol. 24, pp. 509-522, 2002

[7] S. B. Greg Mori, and Jitendra Malik, Efficient Shape Matching Using Shape Contexts, pp. 0-16, 2005.

[8] G. L. Dengsheng Zhang, "A comparative study of fourier descriptors for shape representation and retrieval," in ACCV2002: The 5th Asian Conference on Computer Vision, ed. Melbourne, Australia, 2002

[9] M. L. Zhaozhong Wang, Y.F. Li, Using diagonals of orthogonal projection matrices for affine invariant contour matching, Image and Vision Computing vol. 29, pp. 681-692, 2011

Figure 4. The results of point matching under (a) rotation, (b) scale, (c) shearing, and (d) noise. 